\title{
A canonical form for the design of unknown input sliding mode observers
}

\author{
T. Floquet ${ }^{1}$ and J.P. Barbot ${ }^{2}$ \\ 1 LAGIS UMR CNRS 8146, BP 48, 59651 Villeneuve-d'Ascq, France \\ thierry.floquet@ec-lille.fr \\ 2 ECS, ENSEA, 6 Avenue du Ponceau, 95014 Cergy, France \\ barbot@ensea.fr
}

\subsection{Introduction}

The concept of sliding mode control [11, 27, 32] has been extended to the problem of the state estimation by an observer, both for linear [10, 32] and nonlinear systems $[1,8,28,34]$. Using the same design theory as variable structure control laws, the observer trajectories are constrained to evolve after a finite time on a suitable sliding manifold, by the use of a discontinuous output injection (the sliding manifold is usually given by the difference between the observer and the system output). The sliding motion provides an estimation (asymptotically or in finite time) of the system state. Sliding mode observers have been shown to be efficient in many applications, such as in robotics $[3,21]$, electrical engineering $[6,33]$, chemical reactors [25] or fault detection $[14,18]$.

The problem of designing an observer for a multivariable linear system partially driven by unknown inputs is of great interest. Such a problem arises in systems subject to disturbances or with inaccessible inputs and in many applications such as fault detection and isolation, parameter identification or cryptography. Since, in the sliding mode, the resulting dynamics are insensitive to a class of perturbations and parametric uncertainties, variable structure techniques for robust state reconstruction have been developed in many papers. Observers are designed using canonical forms which are derived under the assumption that some matching conditions are fulfilled by the system.

The main contribution of this chapter is to introduce a constructive algorithm that transforms the system into a new canonical form suitable for the design of finite time sliding mode observers ${ }^{3}$. An interesting property of the algorithm is that it can be performed to estimate the state of some systems

\footnotetext{
${ }^{3}$ Note that the system has to be fully observable for a finite time convergence
} property. 
that do not necessarily satisfy the classical matching conditions involved in the design of sliding mode observers.

This work is organized as follows. Section 1.2 gives a brief overview of robust state reconstruction based on sliding mode observers and the topic of this work. In Section 1.3 the algorithm is developed, which results in a canonical form made of block triangular observable forms. Then in Section 1.4, the state and the unknown inputs are estimated by means of a finite time observer designed under second order sliding mode considerations. An example, that points out the feasibility and the advantages of the proposed method, is given Section 1.5. Throughout the paper, we use the notation:

$$
\Delta(l)=\left(\begin{array}{ccccc}
0 & 1 & 0 & 0 & 0 \\
0 & 0 & 1 & 0 & 0 \\
\vdots & \vdots & \vdots & \ddots & \vdots \\
0 & 0 & 0 & 0 & 1 \\
0 & 0 & 0 & 0 & 0
\end{array}\right) \in \mathbb{R}^{l \times l}
$$

\subsection{Motivation and problem statement}

Motivation:

This chapter is concerned with the design of a sliding mode observer for a linear time-invariant system subject to unknown inputs:

$$
\begin{aligned}
& \dot{x}=A x+B u+D w \\
& y=\left[y_{1} \cdots y_{p_{1}}\right]^{T}=C x, y_{i}=C_{i} x
\end{aligned}
$$

where $x \in \mathbb{R}^{n}$ is the state vector, $y \in \mathbb{R}^{p_{1}}$ is the output vector, $u \in \mathbb{R}^{q}$ represents the known inputs and $w \in \mathbb{R}^{m}$ stands for the bounded, unknown inputs. $A, B, C$ and $D$ are known constant matrices of appropriate dimension. It is supposed that $m \leq p_{1}$ and without loss of generality, it can be assumed that $\operatorname{rank}(C)=p_{1}$ and that $\operatorname{rank}(D)=m$.

The design of asymptotically stable sliding mode observers usually assumes that the system (1.1-1.2) satisfies two structural conditions (see e.g. [11]): the invariant zeros of $\{A, D, C\}$ lie in $\mathbb{C}_{-}$and

$$
\operatorname{rank}(C D)=\operatorname{rank}(D)=m .
$$

Condition (1.3) is called the observer matching condition, and is the analogue of the well-known matching condition [9] for a sliding mode controller to be insensitive to matched perturbations.

Then, there exists a linear change of coordinates that puts the original system into the canonical form given by:

$$
\left\{\begin{array}{l}
\dot{x}_{1}=A_{11} x_{1}+A_{12} y+B_{1} u, \\
\dot{y}=A_{21} x_{1}+A_{22} y+B_{2} u+D_{1} w(t)
\end{array}\right.
$$


A simple observer was originally designed in [32]. In [10], the authors proposed a method that utilizes both linear and discontinuous output error injection. In [30], this method was extended with a Linear Matrix Inequality (LMI) formulation in order to exploit additional degrees of freedom in the choice of the linear and discontinuous gains and to set the poles of the linear part of the observer in a specified region of the complex plane. These observers have also been recently used in the applicative field of fault detection and identification $[12,31]$ where the fault is reconstructed by analyzing the so-called equivalent output injection (which is the counterpart of the equivalent control in the design of sliding mode control).

Remark 1. In [34], an observer was developed for linear uncertain systems without any state transformation, but with a constrained Lyapunov problem to be solved. It was shown in [4] that this problem has a solution if and only if the two structural conditions hold.

Remark 2. It is worth noticing that the same conditions are required for the design of linear unknown input observers, that use the conventional Luenberger procedure (see e.g. [22] and [13] for a comparative study of both approaches).

Other works on sliding mode observers deal with finite time state estimation for observable systems. They are based on the equivalent control approach. The system is assumed to be put in a so-called block triangular observable form:

$$
\left[\begin{array}{c}
\dot{y} \\
\dot{x}_{2} \\
\vdots \\
\dot{x}_{l-1} \\
\dot{x}_{l}
\end{array}\right]=\left[\begin{array}{cccccc}
A_{11} & A_{12} & 0 & 0 & \ldots & 0 \\
A_{21} & A_{22} & A_{23} & 0 & \ldots & 0 \\
\vdots & & & \ddots & & \vdots \\
\vdots & & & & \ddots & A_{l-1, l} \\
A_{l 1} & \ldots & & & \ldots & A_{l, l}
\end{array}\right]\left[\begin{array}{c}
y \\
x_{2} \\
\vdots \\
x_{l-1} \\
x_{l}
\end{array}\right]+\left[\begin{array}{c}
B_{1} \\
B_{2} \\
\vdots \\
B_{l-1} \\
B_{l}
\end{array}\right] u+\left[\begin{array}{c}
0 \\
0 \\
\vdots \\
0 \\
D_{l}
\end{array}\right] w
$$

The estimation of the state is based on a step-by-step procedure using the successive filtered values of equivalent output injections obtained from first order sliding mode observers. One can refer to the works $[15,19,33]$ for linear systems or to $[1,7,8,35]$ for nonlinear systems. Applications can be found in fault detection and isolation [16] or in cryptography [2]. Step-by-step first order sliding mode observers were also successfully implemented in electrical and mechanical systems $[6,26]$.

Such a method is tractable if the unknown inputs appear in the last block of (1.5). For instance, in the simple case of a single output observable system $\left(p_{1}=1\right)$, the following extended observer matching condition should be satisfied (see [27], Chapter 4): 


$$
\operatorname{rank}\left(\begin{array}{c}
C \\
C A \\
\vdots \\
C A^{n-2}
\end{array}\right) D=0 .
$$

Then, under the change of coordinates

$$
\xi=\left[\begin{array}{c}
C \\
C A \\
\vdots \\
C A^{n-1}
\end{array}\right] x,
$$

the system (1.1-1.2) is transformed into

$$
\begin{aligned}
& \dot{\xi}=\Delta(n) \xi+\left[\begin{array}{c}
0 \\
\vdots \\
0 \\
C A^{n}
\end{array}\right] x+\left[\begin{array}{c}
0 \\
\vdots \\
0 \\
C A^{n-1} D
\end{array}\right] w+B u, \\
& y=\left[\begin{array}{llll}
1 & 0 & \cdots & 0
\end{array}\right] \xi
\end{aligned}
$$

and a finite time sliding mode observer can be designed as it will be shown in Section 1.4.1.

\section{Problem statement:}

Here, the aim is to design a sliding mode observer for system (1.1-1.2), leading to the finite time estimation of both state and unknown inputs, even if the previously mentioned matching conditions (1.3) or (1.6) are not satisfied. To this end, a suitable change of coordinates is provided via a constructive algorithm (as a consequence, this algorithm allows to the assessment of whether full state observability is achieved and the possibility to estimate the unknown inputs). Under this transformation, the system is put in a new type of canonical form. This form is an extension of the classical block triangular observable form, and will be called a quasi block triangular observable form. Actually, it is made of a set of block triangular observable forms, but some of them are fed by fictitious outputs. Then, the use of a variable structure observer, and more particularly a step-by-step second order sliding mode observer, ensures the convergence to zero of the observation errors in finite time. The cornerstone of the procedure is the manipulation in a suitable way of all the equivalent output injections, whose analysis leads to the knowledge of the fictitious outputs. Furthermore, this procedure allows the recovery of the unknown inputs in finite time, under the assumption that they are at least bounded piecewise continuous functions. 


\subsection{Output Information Algorithm}

In this Section an algorithm is given that puts the system into a quasi block triangular observable form.

Step 1: Consider the vector of outputs $y^{1} \triangleq C x$.

a. Without loss of generality, reorder the components of $y^{1}$ as follows:

$$
y^{1}=\left[C_{1}^{T} \cdots C_{\eta_{1}}^{T} C_{\eta_{1}+1}^{T} \cdots C_{p_{1}}^{T}\right]^{T} x
$$

so that for $1 \leq j \leq \eta_{1}$ :

$$
C_{j} A^{k} D=0, \text { for all } k \in \mathbb{N},
$$

and for $1 \leq j \leq p_{1}-\eta_{1}$, there exists an integer $r_{j}^{1}$ such that:

$$
\begin{aligned}
C_{\eta_{1}+j} A^{k} D & =0, \text { for all } k<r_{j}^{1}-1 \\
C_{\eta_{1}+j} A^{r_{j}^{1}-1} D & \neq 0 .
\end{aligned}
$$

The outputs $y_{j}^{1}, j \leq \eta_{1}$, are not affected by the unknown inputs.

b. Compute the set of row vectors

$$
\Phi^{1}=\operatorname{span}\left\{C_{1}, \ldots, C_{1} A^{n-1}, C_{2}, \ldots, C_{2} A^{n-1}, \ldots, C_{\eta_{1}}, \ldots, C_{\eta_{1}} A^{n-1}\right\}
$$

and note $\varphi^{1}=\operatorname{rank}\left(\Phi^{1}\right)$.

Define the integers $\varphi_{j}^{1}, 1 \leq j \leq \eta_{1}$, (not uniquely), in such a way that the matrix

$$
I_{1}=\left[\begin{array}{c}
C_{1} \\
\vdots \\
C_{1} A^{\varphi_{1}^{1}-1} \\
\vdots \\
C_{\eta_{1}} \\
\vdots \\
C_{\eta_{1}} A^{\varphi_{\eta_{1}}^{1}-1}
\end{array}\right]
$$

has rank $\varphi^{1}$. One has $\varphi_{1}^{1}+\ldots+\varphi_{\eta_{1}}^{1}=\varphi^{1}$. If $\varphi^{1}=n$, stop the algorithm. Actually, in such a case, the state is not affected by any disturbance, i.e. $D=0$.

c. Compute the set of row vectors

$$
\Upsilon^{1}=\operatorname{span}\left\{C_{\eta_{1}+1}, \ldots, C_{\eta_{1}+1} A^{r_{1}^{1}-1}, \ldots, C_{p_{1}}, \ldots, C_{p_{1}} A^{r_{p_{1}-\eta_{1}}^{1}-1}\right\}
$$

and write $\varphi^{1}+\rho^{1}=\operatorname{rank}\left(\Phi^{1} \cup \Upsilon^{1}\right)$. 
Define the integers $\left(\rho_{1}^{1}, \ldots, \rho_{p_{1}-\eta_{1}}^{1}\right)$ so that (after a possible reordering of the One has $\rho_{1}^{1}+. .+\rho_{p_{1}-\eta_{1}}^{1}=\rho^{1}$.

In order to explain here the motivation of such computations, let us set the $(n \times n)$ matrix $T_{1}=\left[\begin{array}{c}I_{1} \\ D_{1} \\ \Omega_{1}\end{array}\right]$, where $\Omega_{1} \in \mathbb{R}^{\left(n-\varphi^{1}-\rho^{1}\right) \times n}$ is any matrix such that $\operatorname{rank}\left(T_{1}\right)=n$. Under the change of coordinates $\left[\begin{array}{c}\sigma^{1} \\ \chi^{1} \\ \theta^{1}\end{array}\right]=T_{1} x$, the system (1.1-1.2) is transformed in the following form:

$$
\begin{aligned}
\dot{\sigma}_{j}^{1} & =\Delta\left(\varphi_{j}^{1}\right) \sigma_{j}^{1}+\Xi_{j}^{\sigma^{1}} x+B_{j}^{\sigma^{1}} u, 1 \leq j \leq \eta_{1} \\
\dot{\chi}_{j}^{1} & =\Delta\left(\rho_{j}^{1}\right) \chi_{j}^{1}+\Xi_{j}^{\chi^{1}} x+\Theta_{j}^{\chi^{1}} w+B_{j}^{\chi^{1}} u, 1 \leq j \leq p_{1}-\eta_{1} \\
\dot{\theta}^{1} & =P_{1}\left[\begin{array}{c}
\sigma^{1} \\
\chi^{1} \\
\theta^{1}
\end{array}\right]+Q_{1} w+B^{\theta^{1}} u
\end{aligned}
$$

where $\sigma^{1}=\left[\begin{array}{c}\sigma_{1}^{1} \\ \vdots \\ \sigma_{\eta_{1}}^{1}\end{array}\right], \sigma_{j}^{1} \in \mathbb{R}^{\varphi_{j}^{1}}$, and $\chi^{1}=\left[\begin{array}{c}\chi_{1}^{1} \\ \vdots \\ \chi_{p_{1}-\eta_{1}}^{1}\end{array}\right], \chi_{j}^{1} \in \mathbb{R}^{\rho_{j}^{1}}$, and

$$
\Xi_{j}^{\sigma^{1}}=\left[\begin{array}{c}
0 \\
\vdots \\
0 \\
C_{j} A^{\varphi_{j}^{1}}
\end{array}\right]_{\varphi_{j}^{1} \times n}, \Xi_{j}^{\chi^{1}}=\left[\begin{array}{c}
0 \\
\vdots \\
0 \\
C_{\eta_{1}+j} A^{\rho_{j}^{1}}
\end{array}\right]_{\rho_{j}^{1} \times n}, \Theta_{j}^{\chi^{1}}=\left[\begin{array}{c}
0 \\
\vdots \\
0 \\
C_{\eta_{1}+j} A^{\rho_{j}^{1}-1} D
\end{array}\right]_{\rho_{j}^{1} \times m}
$$

and $B_{j}^{\sigma^{1}}, B_{j}^{\chi^{1}}$ and $B^{\theta^{1}}$ are matrices of appropriate dimension. The outputs $y_{j}^{1}$ are the first components of $\sigma_{j}^{1}$, for $1 \leq j \leq \eta_{1}$, and of $\chi_{j}^{1}$, for $1 \leq j \leq p_{1}-\eta_{1}$. The subsystem (1.7-1.8) is in a block triangular observable form similar to (1.5). Thus, as argued in Section 1.2, the whole state can be estimated in finite time using existing step-by-step sliding mode observers if $\varphi^{1}+\rho^{1}=n$. Unfortunately, this condition is not fulfilled by many systems. If $\varphi^{1}+\rho^{1}<n$, 
the main idea of the algorithm is to compute some fictitious outputs ${ }^{4}$ so that the subsystem (1.9) can be put in a quasi block triangular observable form ${ }^{5}$ that allows the estimation of the remaining part of the state $\theta^{1}$. For this: d. Define the matrix

$$
\Gamma_{1}=\left[\begin{array}{c}
C_{\eta_{1}+1} A^{r_{1}^{1}-1} D \\
\vdots \\
C_{p_{1}} A^{r_{p_{1}-\eta_{1}}^{1}-1} D
\end{array}\right]
$$

and let $d_{1}=\operatorname{rank}\left(\Gamma_{1}\right)$. If $d_{1}=p_{1}-\eta_{1}$, stop the algorithm. If $d_{1}<p_{1}-\eta_{1}$, there exists a matrix $\Lambda_{1} \in \mathbb{R}^{p_{2} \times\left(p_{1}-\eta_{1}\right)}$, where $p_{2}=p_{1}-\eta_{1}-d_{1}$, such that $\Lambda_{1} \Gamma_{1}=0$. Let us then define the auxiliary variable:

$$
y^{2}=\Lambda_{1}\left[\begin{array}{c}
C_{\eta_{1}+1} A^{r_{1}^{1}} \\
\vdots \\
C_{p_{1}} A^{r_{p_{1}-\eta_{1}}^{1}}
\end{array}\right] x \triangleq C^{2} x, C^{2}=\left[\begin{array}{c}
C_{1}^{2} \\
\vdots \\
C_{p_{2}}^{2}
\end{array}\right] .
$$

Note that $C^{2}$ is not necessarily full rank.

Step 2: Apply the Output Information Algorithm to the new vector of fictitious outputs $y^{2} \in \mathbb{R}^{p_{2}}$.

a. After possible reordering of the components of $y^{2}$, by analogy with Step 1.a, define the integers $\eta_{2}$ and $r_{j}^{2}, 1 \leq j \leq p_{2}-\eta_{2}$.

b. In the same way as Step 1.b, define the set $\Phi^{2}$, the integer $\varphi^{2}$ (such that $\left.\operatorname{rank}\left(\Phi^{1} \cup \Upsilon^{1} \cup \Phi^{2}\right)=\varphi^{1}+\rho^{1}+\varphi^{2}\right)$, the integers $\varphi_{j}^{2}, 1 \leq j \leq \eta_{2}$, and the related set $I_{2}$ such that $\operatorname{rank}\left[\begin{array}{c}I_{1} \\ D_{1} \\ I_{2}\end{array}\right]=\varphi^{1}+\rho^{1}+\varphi^{2}$. If $\varphi^{1}+\rho^{1}+\varphi^{2}=n$, stop the algorithm.

c. By analogy with Step 1.c, define the sets $\Upsilon^{2}$ and $D_{2}$ and the related integers $\rho^{2}$ and $\left(\rho_{1}^{2}, \ldots, \rho_{p_{2}-\eta_{2}}^{2}\right)$.

If $\varphi^{1}+\rho^{1}+\varphi^{2}+\rho^{2}=n$, or if $\left\{\varphi^{1}+\rho^{1}+\varphi^{2}+\rho^{2}<n\right.$ and $\left.D_{2}=\emptyset\right\}$, stop the algorithm. The second condition means that there is no fictitious output (affected by the unknown inputs) linearly independent from the previous measurements, that could lead to a (even partially) block triangular observable form for the subsystem (1.9).

d. Define the matrix

$$
\Gamma_{2}=\left[\begin{array}{c}
\Gamma_{1} \\
C_{\eta_{2}+1}^{2} A^{r_{1}^{2}-1} D \\
\vdots \\
C_{p_{2}}^{2} A^{r_{p_{2}-\eta_{2}}^{2}-1} D
\end{array}\right]
$$

${ }^{4}$ these fictitious outputs will be made available by analyzing the equivalent output injections of a suitable sliding mode observer, see Section 1.4.

${ }^{5}$ the term quasi is used because some blocks will be fed by fictitious outputs and not by measured outputs. 
and let $d_{2}=\operatorname{rank} \Gamma_{2}$. If $d_{2}<\left(p_{1}-\eta_{1}\right)+\left(p_{2}-\eta_{2}\right)$, one can find a matrix $\Lambda_{2} \in \mathbb{R}^{p_{3} \times\left(\left(p_{1}-\eta_{1}\right)+\left(p_{2}-\eta_{2}\right)\right)}$, where $p_{3}=\left(p_{1}-\eta_{1}\right)+\left(p_{2}-\eta_{2}\right)-d_{2}$, such that $\Lambda_{2} \Gamma_{2}=0$. Then the Output Information Algorithm is applied to the new fictitious outputs

$$
y^{3}=\Lambda_{2}\left[\begin{array}{c}
C_{\eta_{1}+1} A^{r_{1}^{1}} \\
\vdots \\
C_{p_{1}} A^{r_{p_{1}-\eta_{1}}^{1}} \\
C_{\eta_{2}+1}^{2} A^{r_{1}^{2}} \\
\vdots \\
C_{p_{2}}^{2} A^{r_{p_{2}-\eta_{2}}^{2}}
\end{array}\right] x \triangleq C^{3} x .
$$

Repeating this procedure, one has:

Step k: Consider the fictitious output $y^{k} \in \mathbb{R}^{p_{k}}$, defined in Step $k-1$.

a. Define the integers $\eta_{k}$ and $r_{j}^{k}, 1 \leq j \leq p_{k}-\eta_{k}$.

b. Compute the set of row vectors

$$
\Phi^{k}=\operatorname{span}\left\{C_{1}^{k}, \ldots, C_{1}^{k} A^{n-1}, C_{2}^{k}, \ldots, C_{2}^{k} A^{n-1}, \ldots, C_{\eta_{k}}^{k}, \ldots, C_{\eta_{k}}^{k} A^{n-1}\right\}
$$

and write $\sum_{i=1}^{k-1}\left(\varphi^{i}+\rho^{i}\right)+\varphi^{k}=\operatorname{rank}\left(\left(\bigcup_{i=1}^{k-1} \Phi^{i} \cup \Upsilon^{i}\right) \cup \Phi^{k}\right)$.

Define the integers $\varphi_{j}^{k}, 1 \leq j \leq \eta_{k}$ such that

$$
\operatorname{rank}\left[\begin{array}{c}
I_{1} \\
D_{1} \\
\vdots \\
I_{k}
\end{array}\right]=\sum_{i=1}^{k-1}\left(\varphi^{i}+\rho^{i}\right)+\varphi^{k}
$$

where $I_{k}=\left[\left(C_{1}^{k}\right)^{T}, \ldots,\left(C_{1}^{k} A^{\varphi_{1}^{k}-1}\right)^{T}, \ldots,\left(C_{\eta_{k}}^{k}\right)^{T}, \ldots,\left(C_{\eta_{k}}^{k} A^{\varphi_{\eta_{k}}^{k}-1}\right)^{T}\right]^{T}$. If $\sum_{i=1}^{k-1}\left(\varphi^{i}+\rho^{i}\right)+\varphi^{k}=n$, stop the algorithm.

c. Compute the set of row vectors

$$
\Upsilon^{k}=\operatorname{span}\left\{C_{\eta_{k}+1}^{k}, \ldots, C_{\eta_{k}+1}^{k} A^{r_{1}^{k}-1}, \ldots, C_{p_{k}}^{k}, \ldots, C_{p_{k}}^{k} A^{r_{p_{k}-\eta_{k}}^{k}-1}\right\}
$$

and write $\operatorname{rank}\left(\bigcup_{i=1}^{k} \Phi^{i} \cup \Upsilon^{i}\right)=\sum_{i=1}^{k}\left(\varphi^{i}+\rho^{i}\right)$.

Define the integers $\left(\rho_{1}^{k}, \ldots, \rho_{p_{k}-\eta_{k}}^{k}\right)$ in such a way that

$$
\operatorname{rank}\left[\begin{array}{c}
I_{1} \\
D_{1} \\
\vdots \\
I_{k} \\
D_{k}
\end{array}\right]=\sum_{i=1}^{k}\left(\varphi^{i}+\rho^{i}\right)
$$


where

$$
D_{k}=\left[\left(C_{\eta_{k}+1}^{k}\right)^{T}, \ldots,\left(C_{\eta_{k}+1}^{k} A^{\rho_{1}^{k}-1}\right)^{T}, \ldots,\left(C_{p_{k}}^{k}\right)^{T}, \ldots,\left(C_{p_{k}}^{k} A^{\rho_{p_{k}-\eta_{k}}^{k}-1}\right)^{T}\right]^{T} .
$$

If $\sum_{i=1}^{k}\left(\varphi^{i}+\rho^{i}\right)=n$, or if $\left\{\sum_{i=1}^{k}\left(\varphi^{i}+\rho^{i}\right)<n\right.$ and $\left.D_{k}=\emptyset\right\}$, stop the algorithm.

d. Define

$$
\Gamma_{k}=\left[\begin{array}{c}
\Gamma_{k-1} \\
C_{\eta_{k}+1}^{k} A^{r_{1}^{k}-1} D \\
\vdots \\
C_{p_{k}}^{k} A^{r_{p_{k}-\eta_{k}}^{k}-1} D
\end{array}\right]
$$

and let $d_{k}=\operatorname{rank}\left(\Gamma_{k}\right)$. If $d_{k}<\sum_{s=1}^{k}\left(p_{s}-\eta_{s}\right)$, let us set $p_{k+1}=\sum_{s=1}^{k}\left(p_{s}-\eta_{s}\right)-$ $d_{k}$. There exists a matrix $\Lambda_{k} \in \mathbb{R}^{p_{k+1} \times \sum_{s=1}^{k}\left(p_{s}-\eta_{s}\right)}$ such that $\Lambda_{k} \Gamma_{k}=0$. Then a new fictitious output is defined:

$$
y^{k+1}=\Lambda_{k}\left[\begin{array}{c}
C_{\eta_{1}+1} A^{r_{1}^{1}} \\
\vdots \\
C_{p_{1}} A^{r_{p_{1}-\eta_{1}}^{1}} \\
\vdots \\
C_{\eta_{k}+1}^{k} A^{r_{1}^{k}} \\
\vdots \\
C_{p_{k}}^{k} A^{r_{p_{k}-\eta_{k}}^{k}}
\end{array}\right] x \triangleq C^{k+1} x .
$$

Stop the algorithm if:

1. there exists a $\mu \in \mathbb{N}$, such that

$$
\sum_{i=1}^{\mu}\left(\varphi^{i}+\rho^{i}\right)<n \text { and }\left\{d_{\mu}=\sum_{s=1}^{\mu}\left(p_{s}-\eta_{s}\right) \text { or } D_{\mu}=\emptyset\right\}
$$

2. there exists a $k^{\star} \in \mathbb{N}$ such that $\sum_{i=1}^{k^{\star}}\left(\varphi^{i}+\rho^{i}\right)=n$.

One has $\mu, k^{\star} \leq n-p_{1}$. So, the algorithm stops after a number of steps less than $n-p_{1}$. In case 1 , it is not possible to find new variables leading to a quasi block triangular observable form. In case 2 , one can set the following 
nonsingular $(n \times n)$ matrix $T_{k^{\star}}=\left[\begin{array}{c}I_{1} \\ D_{1} \\ \vdots \\ I_{k^{\star}} \\ D_{k^{\star}}\end{array}\right]$. Under the coordinate transformation $\left[\begin{array}{c}\sigma^{1} \\ \chi^{1} \\ \vdots \\ \sigma^{k^{\star}} \\ \chi^{k^{\star}}\end{array}\right]=T_{k^{\star}} x$ where, for $1 \leq i \leq k^{\star}, \sigma^{i}=\left[\begin{array}{c}\sigma_{1}^{i} \\ \vdots \\ \sigma_{\eta_{i}}^{i}\end{array}\right], \sigma_{j}^{i} \in \mathbb{R}^{\varphi_{j}^{i}}$, and $\chi^{i}=\left[\begin{array}{c}\chi_{1}^{i} \\ \vdots \\ \chi_{p_{i}-\eta_{i}}^{i}\end{array}\right], \chi_{j}^{i} \in \mathbb{R}^{\rho_{j}^{i}}$, the system (1.1-1.2) becomes:

$$
\begin{gathered}
\dot{\sigma}_{j}^{i}=\Delta\left(\varphi_{j}^{i}\right) \sigma_{j}^{i}+\Xi_{j}^{\sigma^{i}} x+B_{j}^{\sigma^{i}} u, 1 \leq j \leq \eta_{i} \\
\dot{\chi}_{j}^{i}=\Delta\left(\rho_{j}^{i}\right) \chi_{j}^{i}+\Xi_{j}^{\chi^{i}} x+\Theta_{j}^{\chi^{i}} w+B_{j}^{\chi^{i}} u, 1 \leq j \leq p_{i}-\eta_{i} \\
\Xi_{j}^{\sigma^{i}}=\left[\begin{array}{c}
0 \\
\vdots \\
0 \\
C_{j} A^{\varphi_{j}^{i}}
\end{array}\right]_{\varphi_{j}^{i} \times n}, \Xi_{j}^{\chi^{i}}=\left[\begin{array}{c}
0 \\
\vdots \\
0 \\
C_{\eta_{i}+j} A^{\rho_{j}^{i}}
\end{array}\right]_{\rho_{j}^{i} \times n}, \Theta_{j}^{\chi^{i}}=\left[\begin{array}{c}
0 \\
\vdots \\
0 \\
C_{\eta_{i}+j} A_{\rho_{j}^{i}-1}{ }_{\rho_{j}^{i} \times m}
\end{array}\right.
\end{gathered}
$$

$B_{i, j}^{\sigma^{i}}$ and $B_{i, j}^{\chi^{i}}$ are a $\left(\varphi_{j}^{i} \times q\right)$ and a $\left(\rho_{j}^{i} \times q\right)$-matrix, respectively. Thus, the system is put in quasi block triangular observable form.

\subsection{Observer design}

\subsubsection{Sliding mode observer for a single triangular observable form}

In this part, a step-by-step sliding mode observer is designed for a system described by the following triangular form:

$$
\dot{\xi}=\Delta(l) \xi+\left[\begin{array}{c}
0 \\
\vdots \\
0 \\
M
\end{array}\right] z+B u, y=\left[\begin{array}{llll}
1 & 0 & \cdots & 0
\end{array}\right] \xi
$$

where $\xi=\left[\xi_{1} \cdots \xi_{l}\right]^{T} \in \mathbb{R}^{l},(l>1)$ is the state vector, $y \in \mathbb{R}$ is the output vector, $u$ is the input vector and $z \in \mathbb{R}^{m}$ stands for some state variables and unknown inputs with $M \in \mathbb{R}^{1 \times m}$. Let us assume that the system is 
Bounded Input Bounded State (BIBS) and that $z$ and its first time derivative are bounded, i.e.:

$$
\begin{aligned}
\left|\xi_{i}\right| & <d_{i}, i=1, \ldots, l \\
\|z\| & <K \\
\|\dot{z}\| & <K^{\prime}
\end{aligned}
$$

where $d_{i}, K$ and $K^{\prime}$ are some known positive scalars.

Most of sliding mode observer designs for (1.12) are based on a step-by-step procedure using successive filtering values of the so-called equivalent output injections obtained from recursive first order sliding mode observers. However, the approximation of the equivalent information injections by low pass filters at each step may introduce some delays that could lead to inaccurate estimates or to instability for high order systems ${ }^{6}$. To overcome this problem, here it is proposed to replace the discontinuous first order sliding mode output injection by a continuous second order sliding mode one. The observer is built as follows:

$$
\left\{\begin{array}{l}
\frac{d \hat{\xi}_{1}}{d t}=\nu\left(y-\hat{\xi}_{1}\right) \\
\frac{d \hat{\xi}_{2}}{d t}=E_{1} \nu\left(\tilde{\xi}_{2}-\hat{\xi}_{2}\right) \\
\vdots \\
\frac{d \hat{\xi}_{l-1}}{d t}=E_{l-2} \nu\left(\tilde{\xi}_{l-1}-\hat{\xi}_{l-1}\right) \\
\frac{d \hat{\xi}_{l}}{d t}=E_{l-1} \nu\left(\tilde{\xi}_{l}-\hat{\xi}_{l}\right)
\end{array}+B u\right.
$$

with

$$
\begin{aligned}
& \tilde{\xi}_{1}=y \\
& \tilde{\xi}_{j}=\nu\left(\tilde{\xi}_{j-1}-\hat{\xi}_{j-1}\right), 2 \leq j \leq l
\end{aligned}
$$

where the continuous output injection $\nu$ is given by the so-called super twisting algorithm [17, 23]:

$$
\left\{\begin{array}{l}
\nu(s)=\varphi(s)+\lambda_{s}|s|^{\frac{1}{2}} \operatorname{sign}(s) \\
\dot{\varphi}(s)=\alpha_{s} \operatorname{sign}(s) \\
\lambda_{s}, \alpha_{s}>0
\end{array} .\right.
$$

Conditions on choice of $\lambda_{s}$ and $\alpha_{s}$ will be explained later. The function $E_{i}$ is equal to 1 if all the previous error components have converged to zero, else $E_{i}=0$. This is an anti-peaking structure [29]. Indeed, with this particular function, the manifolds are reached one by one. At each step, a subdynamics of dimension one is obtained and consequently no peaking phenomena appear. Denoting $e=\xi-\hat{\xi}$, the error dynamics is given by:

\footnotetext{
${ }^{6}$ In [20], the implementation of such filters was studied and a relation between the estimation accuracy, the filter time constants and the sampling time was given.
} 


$$
\left\{\begin{array}{l}
\dot{e}_{1}=\xi_{2}-\nu\left(y-\hat{\xi}_{1}\right) \\
\dot{e}_{2}=\xi_{3}-E_{1} \nu\left(\tilde{\xi}_{2}-\hat{\xi}_{2}\right) \\
\vdots \\
\dot{e}_{l-1}=\xi_{l}-E_{l-2} \nu\left(\tilde{\xi}_{l-1}-\hat{\xi}_{l-1}\right) \\
\dot{e}_{l}=M z-E_{l-1} \nu\left(\tilde{\xi}_{l}-\hat{\xi}_{l}\right)
\end{array}\right.
$$

First step: Before sliding mode on $e_{1}=0$, one has:

$$
\left\{\begin{array}{l}
\dot{e}_{1}=\xi_{2}-\nu\left(e_{1}\right) \\
\dot{e}_{2}=\xi_{3} \\
\vdots \\
\dot{e}_{l-1}=\xi_{l} \\
\dot{e}_{l}=M z
\end{array}\right.
$$

The super twisting algorithm has been developed for systems with relative degree one with respect to the input as in $\dot{e}_{1}=\xi_{2}-\nu\left(e_{1}\right)$. The second time derivative of $e_{1}$ is given by:

$$
\ddot{e}_{1}=\dot{\xi}_{2}-\dot{\nu}\left(e_{1}\right)=\xi_{3}-\frac{1}{2} \lambda_{e_{1}} \dot{e}_{1}\left|e_{1}\right|^{\frac{1}{2}}-\alpha_{e_{1}} \operatorname{sign}\left(e_{1}\right) .
$$

Note that the discontinuity is embedded in the higher derivatives of the error variables, so that they are less affected by the chattering phenomenon. It was shown in [24] that sufficient conditions for the finite time convergence on the second order sliding set $\left\{e_{1}=\dot{e}_{1}=0\right\}$ are:

$$
\begin{aligned}
& \alpha_{e_{1}}>d_{3}, \\
& \lambda_{e_{1}}^{2}>4 d_{3} \frac{\alpha_{e_{1}}+d_{3}}{\alpha_{e_{1}}-d_{3}} .
\end{aligned}
$$

Then, the equivalent dynamics provides a continuous estimation (without any introduction of low pass filters) in finite time of $\xi_{2}$ since $\nu\left(e_{1}\right)=\xi_{2}$ on $\dot{e}_{1}=0$. This also implies that $\tilde{\xi}_{2}-\hat{\xi}_{2}=e_{2}$.

Second step: after a finite time $T_{1},(1.15)$ becomes:

$$
\left\{\begin{array}{l}
\dot{e}_{1}=0 \\
\dot{e}_{2}=\xi_{3}-\nu\left(e_{2}\right) \\
\vdots \\
\dot{e}_{l-1}=\xi_{l} \\
\dot{e}_{l}=M z
\end{array}\right.
$$

and one gets the finite time estimation of $\xi_{3}$.

Following the same scheme till the $(l-1)$-th step, the observer (1.13) provides an estimation of the whole state $\xi$ in finite time. 
l-th step: (1.15) is given by:

$$
\left\{\begin{array}{l}
\dot{e}_{1}=\ldots=\dot{e}_{l-1}=0 \\
\dot{e}_{l}=M z-\nu\left(e_{l}\right)
\end{array}\right.
$$

Thus, with the following choice of gains,

$$
\begin{aligned}
& \alpha_{e_{l}}>\|M\| K^{\prime}, \\
& \lambda_{e_{l}}^{2}>4\|M\| K^{\prime} \frac{\alpha_{e_{l}}+\|M\| K^{\prime}}{\alpha_{e_{l}}-\|M\| K^{\prime}},
\end{aligned}
$$

one obtains directly, on the sliding manifold $e_{l}=\dot{e}_{l}=0$, a continuous approximation of the unknown variables:

$$
\nu\left(e_{l}\right)=M z
$$

The step-by-step observer achieves finite time convergence of the state components. This property is often desirable in the framework of observation, fault detection or identification problems, and in general to solve in finite time the problem of left inversion and state observation.

\subsubsection{A finite time observer for the quasi block triangular observable form}

It can be seen that, after transformation, the system (1.10)-(1.11) is made of a set of triangular forms similar to (1.12). Thus, for $1 \leq i \leq k^{\star}$, the observer is described by:

$$
\begin{array}{r}
\frac{d}{d t} \hat{\sigma}_{j}^{i}=\Xi_{j}^{\sigma^{i}} \hat{x}+B_{j}^{\sigma^{i}} u+\bar{\nu}\left(y_{j}^{i}, \hat{\sigma}_{j}^{i}, \tilde{\sigma}_{j}^{i}\right), 1 \leq j \leq \eta_{i} \\
\frac{d}{d t} \hat{\chi}_{j}^{i}=\Xi_{j}^{\chi^{i}} \hat{x}+B_{j}^{\chi^{i}} u+\bar{\nu}\left(y_{\eta_{i}+j}^{i}, \hat{\chi}_{j}^{i}, \tilde{\chi}_{j}^{i}\right), 1 \leq j \leq p_{i}-\eta_{i}
\end{array}
$$

where $\hat{x}=T_{k^{\star}}^{-1}\left[\begin{array}{c}\hat{\sigma}^{1} \\ \hat{\chi}^{1} \\ \vdots \\ \hat{\sigma}^{k^{\star}} \\ \hat{\chi}^{k^{\star}}\end{array}\right]$, and where

$$
\bar{\nu}\left(y_{j}^{i}, \hat{\sigma}_{j}^{i}, \tilde{\sigma}_{j}^{i}\right)=\left[\begin{array}{c}
\nu\left(y_{j}^{i}-\hat{\sigma}_{j, 1}^{i}\right) \\
E_{2} \nu\left(\tilde{\sigma}_{j, 2}^{i}-\hat{\sigma}_{j, 2}^{i}\right) \\
\vdots \\
E_{\varphi_{j}^{i}} \nu\left(\tilde{\sigma}_{j, \varphi_{j}^{i}}^{i}-\hat{\sigma}_{j, \varphi_{j}^{i}}^{i}\right)
\end{array}\right]
$$

and 


$$
\begin{aligned}
& \bar{\nu}\left(y_{\eta_{i}+j}^{i}, \hat{\chi}_{j}^{i}, \tilde{\chi}_{j}^{i}\right)=\left[\begin{array}{c}
\nu\left(y_{\eta_{i}+j}^{i}-\hat{\chi}_{j, 1}^{i}\right) \\
E_{2} \nu\left(\tilde{\chi}_{j, 2}^{i}-\hat{\chi}_{j, 2}^{i}\right) \\
\vdots \\
E_{\rho_{j}^{i} \nu}\left(\tilde{\chi}_{j, \rho_{j}^{i}}^{i}-\hat{\chi}_{j, \rho_{j}^{i}}^{i}\right)
\end{array}\right] . \\
& \tilde{\sigma}_{j, 1}^{i}=y_{j}^{i} \\
& \tilde{\sigma}_{j, k}^{i}=\nu\left(\tilde{\sigma}_{j, k-1}^{i}-\hat{\sigma}_{j, k-1}^{i}\right) \text { for } k=2, \ldots, \varphi_{j}^{i}, \\
& \tilde{\chi}_{j, 1}^{i}=y_{\eta_{i}+j}^{i} \\
& \tilde{\chi}_{j, k}^{i}=\nu\left(\tilde{\chi}_{j, k-1}^{i}-\hat{\chi}_{j, k-1}^{i}\right) \text { for } k=2, \ldots, \rho_{j}^{i} .
\end{aligned}
$$

$\hat{\sigma}_{j, k}^{1}$ and $\hat{\chi}_{j, k}^{1}$ are the $k$-th components of $\hat{\sigma}_{j}^{1}$ and $\hat{\chi}_{j}^{1}$, respectively and $\nu$ is the continuous output injection defined in (1.14). The following hypothesis are required:

H1. the system is BIBS: Bounded Input Bounded State,

H2. the term $w$ is bounded and is Lebesgue integrable.

The following proposition summarizes the results given in this work:

Proposition 1. Assume that there exists a $k^{\star} \in \mathbb{N}$ such that $\sum_{i=1}^{k^{\star}}\left(\varphi^{i}+\rho^{i}\right)=n$ and that hypothesis $H 1$ and $H 2$ are satisfied. Then:

(i) $\operatorname{rank}\left(\Gamma_{k^{*}}\right)=m$,

(ii) the state is observable and the unknown inputs can be estimated, both of them in finite time by using the step-by-step sliding mode observer (1.16)(1.17).

\section{Proof:}

(i) From the definitions of $I_{i}$ and $D_{i}$, and since $\sum_{i=1}^{k^{\star}}\left(\varphi^{i}+\rho^{i}\right)=n$ :

$$
\begin{aligned}
& \operatorname{rank}\left(\Gamma_{k^{\star}}\right)=\operatorname{rank}\left[\begin{array}{c}
C_{\eta_{1}+1} A^{r_{1}^{1}-1} \\
\vdots \\
C_{p_{1}} A^{r_{p_{1}-\eta_{1}}^{1}-1} \\
\vdots \\
C_{\eta_{k^{\star}}+1}^{k^{\star}} A^{r_{1}^{k^{\star}}-1} \\
\vdots \\
C_{p_{k^{\star}}}^{k^{\star}} A^{r_{p_{k^{\star}}}^{k^{\star}}}
\end{array}\right] D \\
& =\operatorname{rank}\left[\begin{array}{c}
I_{1} \\
D_{1} \\
\vdots \\
I_{k^{\star}} \\
D_{k^{\star}}
\end{array}\right] D=m .
\end{aligned}
$$


(ii) The convergence of the observer is proved hereafter.

Convergence of the state variables

We first design an observer for the subsystems given by $i=1$, i.e. with the available measurement vector $y_{j}^{1}=C_{j} x, 1 \leq j \leq p_{1}$. According to (1.161.17), the error dynamics $\bar{\sigma}^{1}=\sigma^{1}-\hat{\sigma}^{1}$ and $\bar{\chi}^{1}=\chi^{1}-\hat{\chi}^{1}$ are described by the following differential equations:

$$
\begin{gathered}
\frac{d}{d t} \bar{\sigma}_{j}^{1}=\Delta\left(\varphi_{j}^{1}\right) \sigma_{j}^{1}+\Xi_{j}^{\sigma^{1}}(x-\hat{x})-\bar{\nu}\left(y_{j}^{1}, \hat{\sigma}_{j}^{1}, \tilde{\sigma}_{j}^{1}\right), \\
\frac{d}{d t} \bar{\chi}_{j}^{1}=\Delta\left(\rho_{j}^{1}\right) \chi_{j}^{1}+\Xi_{j}^{\chi^{1}}(x-\hat{x})+\Theta_{j}^{\chi^{1}} w-\bar{\nu}\left(y_{\eta_{1}+j}^{1}, \hat{\chi}_{j}^{1}, \tilde{\chi}_{j}^{1}\right) .
\end{gathered}
$$

Every subsystem in (1.18) and (1.19) is in a form similar to (1.15). Thus the variables $\bar{\sigma}^{1}$ and $\bar{\chi}^{1}$ tend to zero in a finite time $t_{1}$. Particularly, a sliding mode occurs on the manifolds $\bar{\chi}_{j, \rho_{j}^{1}}^{1}=0,1 \leq j \leq p_{1}-\eta_{1}$, and the analysis of the resulting equivalent dynamics on the sliding surfaces (obtained by writing that $\left.\frac{d}{d t} \bar{\chi}_{j, \rho_{j}^{1}}^{1}=0\right)$ provides the following $\left(p_{1}-\eta_{1}\right)$ algebraic equations:

$$
\nu\left(\bar{\chi}_{j, \rho_{j}^{1}}^{1}\right)=C_{\eta_{1}+j} A^{\rho_{j}^{1}}(x-\hat{x})+C_{\eta_{1}+j} A^{\rho_{j}^{1}-1} D w .
$$

It can be noted that in the subsequent subsystems $\left(2 \leq i \leq k^{*}\right)$, the information injections are not directly available since they are linear combination of some unknown states. Nevertheless, the equivalent control methodology can be used to obtain additional information about $y^{2}$ from equations (1.20). Let us introduce the auxiliary variable

$$
\tilde{y}^{1}=\Lambda_{1}\left[\begin{array}{c}
C_{\eta_{1}+1} A^{r_{1}^{1}-1} \\
\vdots \\
C_{p_{1}} A^{r_{p_{1}-\eta_{1}}-1}
\end{array}\right] x .
$$

Note that from the construction of $\Upsilon^{1}, I_{1}$ and $D_{1}$ in Step 1.c. of the algorithm, one can write that

$$
\left[\begin{array}{c}
C_{\eta_{1}+1} A^{r_{1}^{1}-1} \\
\vdots \\
C_{p_{1}} A^{r_{p_{1}-\eta_{1}}-1}
\end{array}\right]=G_{1}\left[\begin{array}{c}
I_{1} \\
D_{1}
\end{array}\right]
$$

where $G_{1}$ is a matrix of appropriate dimension. Thus $\tilde{y}^{1}$ can be rewritten as

$$
\tilde{y}^{1}=\Lambda_{1} G_{1}\left[\begin{array}{c}
I_{1} \\
D_{1}
\end{array}\right] x=\Lambda_{1} G_{1}\left[\begin{array}{c}
\sigma^{1} \\
\chi^{1}
\end{array}\right]
$$

and represents available information. Then, the following second order sliding mode observer can be designed 


$$
\frac{d \varsigma^{1}}{d t}=\nu\left(\tilde{y}^{1}-\varsigma^{1}\right)
$$

The dynamics of the error $\varepsilon=\tilde{y}^{1}-\varsigma^{1}$ is given by

$$
\begin{aligned}
\dot{\varepsilon} & =\Lambda_{1}\left[\begin{array}{c}
C_{\eta_{1}+1} A^{r_{1}^{1}-1} \\
\vdots \\
C_{p_{1}} A_{p_{1}-\eta_{1}}^{r_{1}^{1}}-1
\end{array}\right](A x+D w)-\nu\left(\tilde{y}^{1}-\varsigma^{1}\right) \\
& =C^{2} x-\nu(\varepsilon) .
\end{aligned}
$$

Thus, with a suitable choice of the gains of the output injection $\nu(\varepsilon)$, a sliding mode occurs on, $\varepsilon=\dot{\varepsilon}=0$. Then the fictitious output is known after a finite time since:

$$
\nu(\varepsilon)=C^{2} x=y^{2} .
$$

Remark 3. If $\rho_{j}^{1}=r_{j}^{1}$ for all $1 \leq j \leq p_{1}-\eta_{1}, y^{2}$ can be obtained without any additional observer. Indeed, equations (1.20) become:

$$
\begin{aligned}
\nu^{1} & =\left[\begin{array}{c}
\nu\left(\bar{\chi}_{1, \rho_{1}^{1}}^{1}\right) \\
\vdots \\
\nu\left(\bar{\chi}_{p_{1}-\eta_{1}, \rho_{p_{1}-\eta_{1}}^{1}}^{1}\right)
\end{array}\right] \\
& =\left[\begin{array}{c}
C_{\eta_{1}+1} A^{r_{1}^{1}} \\
\vdots \\
C_{p_{1}} A^{r_{p_{1}-\eta_{1}}^{1}}
\end{array}\right](x-\hat{x})+\Gamma_{1} w .
\end{aligned}
$$

Since $\Lambda_{1} \Gamma_{1}=0$ and from the definition of $y^{2}$, one has:

$$
\Lambda_{1} \nu^{1}=y^{2}-\hat{y}^{2} .
$$

The fictitious output $y^{2}$ is henceforth available and repeating the same procedure, for $i=2$, in the observer (1.16-1.17), one obtains an estimation of $\sigma^{2}$ and $\chi^{2}$ after a finite time $t_{2}$.

Following the same scheme step by step, one gets $\sigma^{i}$ and $\chi^{i}, 1 \leq i \leq k^{\star}$ in a finite time $t_{k^{\star}}$. Thus the whole state of the system has been observed.

\section{Estimation of the unknown inputs}

After $t_{k^{\star}}$, additional information can be collected from the equivalent dynamics on the sliding surfaces, namely all the equivalent information injections, for $1 \leq i \leq k^{\star}$ :

$$
\nu\left(\bar{\chi}_{j, \rho_{j}^{i}}^{i}\right)=C_{\eta_{i}+j} A^{\rho_{j}^{i}-1} D w, 1 \leq j \leq p_{i}-\eta_{i} .
$$

This can be written in compact form $V=\Theta^{D} w$, where 


$$
\Theta^{D}=\left[\begin{array}{c}
\Theta_{1}^{D} \\
\vdots \\
\Theta_{k^{\star}}^{D}
\end{array}\right], \Theta_{i}^{D}=\left[\begin{array}{c}
C_{\eta_{i}+1} A^{\rho_{1}^{i}-1} D \\
\vdots \\
C_{p_{i}} A^{\rho_{p_{i}-\eta_{i}}^{i}-1} D
\end{array}\right] \text {. }
$$

The vector $V \in \mathbb{R}^{\sum_{i=1}^{k^{\star}}\left(p_{i}-\eta_{i}\right)}$ has the form:

$$
V=\left[\begin{array}{c}
\nu^{1} \\
\vdots \\
\nu^{\star}
\end{array}\right], \nu^{i}=\left[\begin{array}{c}
\nu\left(\bar{\chi}_{1, \rho_{1}^{i}}^{i}\right) \\
\vdots \\
\nu\left(\bar{\chi}_{p_{i}-\eta_{i}, \rho_{p_{i}-\eta_{i}}^{i}}^{i}\right)
\end{array}\right]
$$

and is an available information that is computable online. Following the same arguments as in Proposition 1, one has $\operatorname{rank}\left(\Theta^{D}\right)=m$. Thus, the relations (1.22) provide a finite time estimation $\hat{w}$ of the unknown inputs $w$ :

$$
\hat{w}=\left(\Theta^{D}\right)^{+} V
$$

where $\left(\Theta^{D}\right)^{+}$is the pseudo-inverse of $\Theta^{D}$.

\subsection{Example}

By way of illustration, let us consider the system described by the following differential equations:

$$
\begin{aligned}
& \dot{x}=\left[\begin{array}{ccccc}
-2 & -1 & 0 & 1 & 0 \\
0 & -1 & 0 & 0 & 1 \\
1 & 0 & -1 & 0 & 0 \\
0 & -1 & -2 & 0 & 1 \\
2 & 0 & 0 & 0 & -1
\end{array}\right] x+\left[\begin{array}{cc}
0 & 0 \\
0 & -1 \\
1 & 0 \\
0 & -2 \\
-2 & 1
\end{array}\right] w \\
& y=\left[\begin{array}{lllll}
1 & 0 & 0 & 0 & 0 \\
0 & 1 & 0 & 0 & 0
\end{array}\right] x=\left[\begin{array}{l}
C_{1} \\
C_{2}
\end{array}\right] x
\end{aligned}
$$

Note that $\operatorname{rank}(C D) \neq \operatorname{rank}(D)$, and that the system can not be put in a form similar to (1.5) suitable for the design of classical step-by-step sliding mode observers. Applying Step 1 of the algorithm, one can check that $I_{1}=0$ and:

$$
\begin{gathered}
D_{1}=\left[\begin{array}{c}
C_{1} \\
C_{1} A \\
C_{2}
\end{array}\right] \\
\Gamma_{1}=\left[\begin{array}{c}
C_{1} A D \\
C_{2} D
\end{array}\right]=\left[\begin{array}{ll}
0 & -1 \\
0 & -1
\end{array}\right] .
\end{gathered}
$$


Thus $\Lambda_{1}=[-11]$ and one can choose the fictitious output as $y^{2}=$ $\Lambda_{1}\left[\begin{array}{c}C_{1} A^{2} \\ C_{2} A\end{array}\right] x=C_{1}^{2} x \in \mathbb{R}$. Step 2 implies that $I_{2}=0$ and $D_{2}=\left[\begin{array}{c}C_{1}^{2} \\ C_{1}^{2} A\end{array}\right]$. Since

$$
\operatorname{rank}(T)=\operatorname{rank}\left[\begin{array}{c}
D_{1} \\
D_{2}
\end{array}\right]=5,
$$

one can define the state transformation $z=T x$. This gives

$$
\begin{gathered}
\dot{z}=\left\{\begin{array}{cc}
z_{2} \\
-4 z_{1}-2 z_{2}+z_{3}+2 z_{4}+z_{5} & -w_{2} \\
-4 z_{1}-2 z_{2}+z_{3}+3 z_{4}+z_{5} & -w_{2} \\
z_{5} & \\
6 z_{1}+6 z_{2}-2 z_{3}-5 z_{4}-4 z_{5} & -2 w_{1}+w_{2}
\end{array}\right. \\
y=\left[z_{1} z_{3}\right]^{T}
\end{gathered}
$$

Then, the observer is designed as follows:

$$
\begin{aligned}
& \frac{d \hat{z}_{1}}{d t}=\nu\left(y_{1}-\hat{z}_{1}\right) \\
& \frac{d \hat{z}_{2}}{d t}=-4 z_{1}-2 \hat{z}_{2}+z_{3}+2 \hat{z}_{4}+\hat{z}_{5}+E\left(y_{1}-\hat{z}_{1}\right) \nu\left(\tilde{z}_{2}-\hat{z}_{2}\right) \\
& \frac{d \hat{z}_{3}}{d t}=-4 z_{1}-2 \hat{z}_{2}+z_{3}+3 \hat{z}_{4}+\hat{z}_{5}+\nu\left(y_{2}-\hat{z}_{3}\right) \\
& \frac{d \hat{z}_{4}}{d t}=\nu\left(y^{2}-\hat{z}_{4}\right) \\
& \frac{d \hat{z}_{5}}{d t}=6 z_{1}+6 \hat{z}_{2}-2 z_{3}-5 \hat{z}_{4}-4 \hat{z}_{5}+E\left(y^{2}-\hat{z}_{4}\right) \nu\left(\tilde{z}_{5}-\hat{z}_{5}\right)
\end{aligned}
$$

The function $E$ is defined by

$$
E(s)=\left\{\begin{array}{l}
0 \text { if } s \neq 0 \\
1 \text { otherwise }
\end{array}\right.
$$

and

$$
\begin{aligned}
& \tilde{z}_{2}=\nu\left(y_{1}-\hat{z}_{1}\right) \\
& \tilde{z}_{5}=\nu\left(y^{2}-\hat{z}_{4}\right)
\end{aligned}
$$

Let us first consider the subsystems (1.23-1.24) and (1.25) (where the information injection is available), which are in block observable triangular form. The error dynamics $e_{i}=z_{i}-\hat{z}_{i}$ can be described by:

$$
\begin{aligned}
& \dot{e}_{1}=z_{2}-\nu\left(e_{1}\right) \\
& \dot{e}_{2}=-2 e_{2}-2 e_{4}+e_{5}-w_{2}-E\left(y_{1}-\hat{z}_{1}\right) \nu\left(\tilde{z}_{2}-\hat{z}_{2}\right) \\
& \dot{e}_{3}=-2 e_{2}-3 e_{4}+e_{5}-w_{2}-\nu\left(e_{3}\right)
\end{aligned}
$$


From the methodology given in section 1.4.1, one recover in a finite time $t_{1}$ the state variables $z_{1}, z_{2}$ and $z_{3}$ and the equivalent output injections:

$$
\begin{aligned}
& \nu\left(e_{2}\right)=2 e_{4}+e_{5}-w_{2} \\
& \nu\left(e_{3}\right)=3 e_{4}+e_{5}-w_{2}
\end{aligned}
$$

It can be seen that the quantity $y^{2}-\hat{z}_{4}=e_{4}=\nu\left(e_{3}\right)-\nu\left(e_{2}\right)$ is henceforth available on the sliding manifold $\left\{e_{2}=0 \cap e_{3}=0\right\}$. Thus, after $t_{1}$, the second part of the observer can be used to reconstruct the remaining variables:

$$
\begin{aligned}
& \dot{e}_{4}=z_{5}-\nu\left(e_{4}\right) \\
& \dot{e}_{5}=5 e_{4}-4 e_{5}-2 w_{1}+w_{2}+E\left(y^{2}-\hat{z}_{4}\right) \nu\left(\tilde{z}_{5}-\hat{z}_{5}\right)
\end{aligned}
$$

Again, we have a step-by-step observer. Thus, the errors $e_{4}$ and $e_{5}$ converge to zero in finite time and one has the corresponding equivalent information injection

$$
\nu\left(e_{5}\right)=2 w_{1}-w_{2} .
$$

The disturbance $w$ is also estimated in finite time since:

$$
\begin{aligned}
& w_{1}=-\frac{1}{2}\left(\nu\left(e_{2}\right)+\nu\left(e_{5}\right)\right) \\
& w_{2}=-\nu\left(e_{2}\right)=-\nu\left(e_{3}\right)
\end{aligned}
$$

Simulations were performed for the observer (1.23-1.27). In practise, the function $E$ is set as follows:

$$
E(s)=\left\{\begin{array}{l}
\varepsilon \text { if } s \neq 0 \\
1 \text { otherwise }
\end{array}\right.
$$

where $\varepsilon$ is a small positive constant. Figure 1.1 depicts the unmeasured states and their estimates. It can be seen that the observer converges quite fast and with good accuracy, as it can be seen in Figure 1(d) in the case of the error between $x_{5}$ and its estimate. Figure 1.2 illustrates the fact that the unknown inputs (that have been chosen as two sine functions) are recovered satisfactory. 


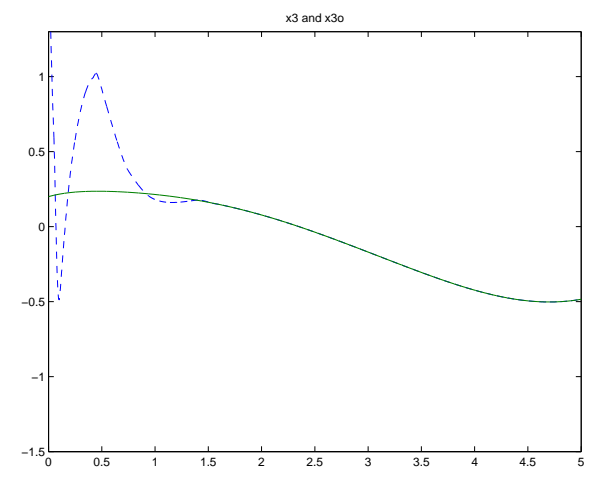

(a)

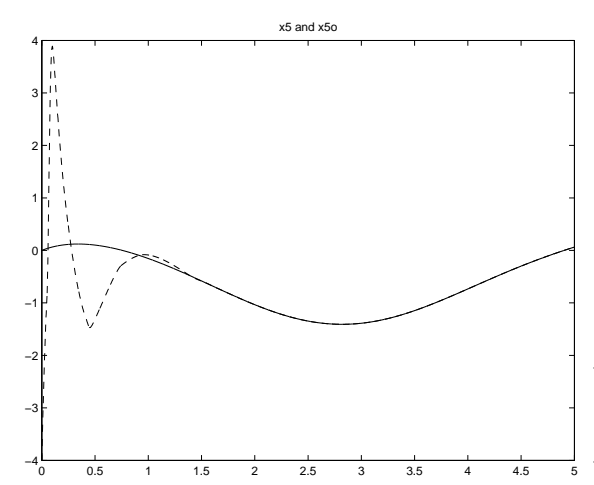

(c)

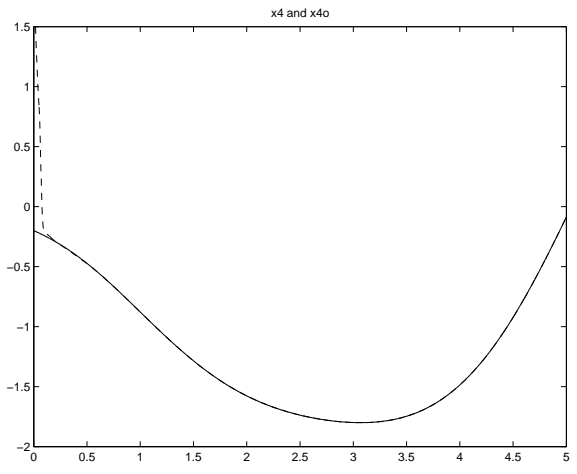

(b)

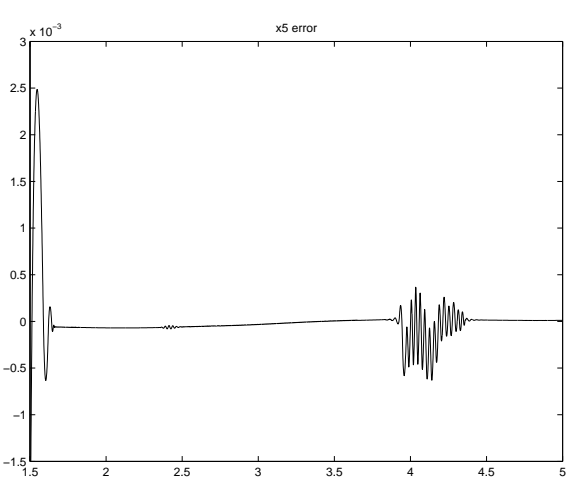

(d)

Fig. 1.1. State (solid line) and estimation (dotted line)

\subsection{Concluding remarks}

In this work, a new approach to solve the problem of designing an unknown input observer for linear systems has been developed. An algorithm was given in order to find a suitable change of coordinates for the design of a step-by step second order sliding mode observer. This observer provides, by using the equivalent output injections, a finite time estimation of both state and unknown inputs. Since the observer is based on second order sliding mode algorithms, the equivalent output injections are obtained in a continuous way without any use of low pass filters. 

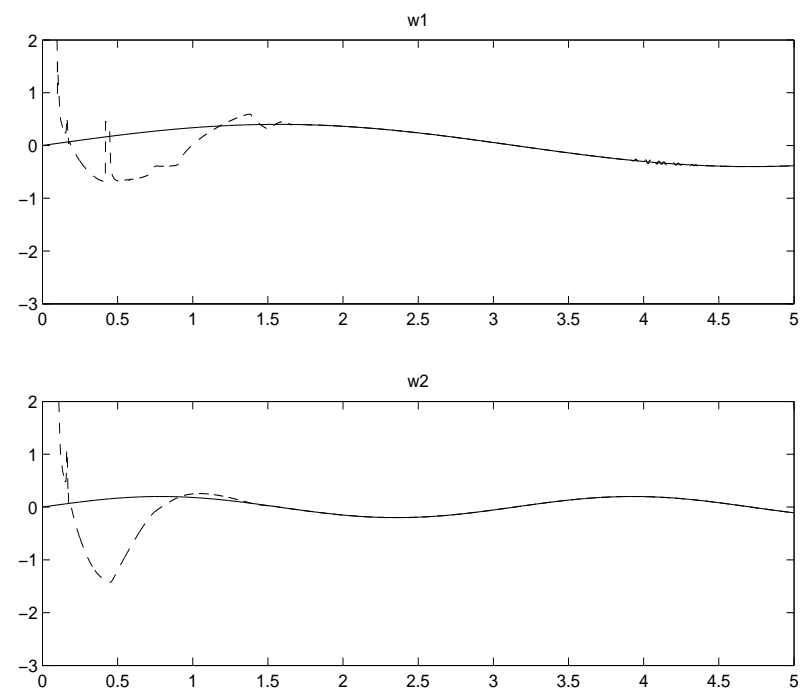

Fig. 1.2. Unknown input (solid line) and its estimation (dotted line)

It is important to note that this algorithm gives a solution to the observation problem of a class of systems subject to unknown inputs that do not necessarily satisfy the standard matching condition $\operatorname{rank}(C D)=\operatorname{rank}(D)$ as it has been seen in the example. It can also be said that the method given here extends other results in the area of sliding mode observers. Indeed, the first step of the algorithm takes into account existing methods that require that the systems can be put in a block observable form where the unknown inputs appear in the last block.

The Output Information Algorithm is a constructive one, and one has to go through all the algorithm to know if it can (or can not) provide a suitable change of coordinates for the design of a step-by-step sliding mode observer. The problem of finding structural conditions (if any) on the original system stating, a priori, whether this method might work or not is an open one. Future research is also concerned with the case of nonlinear systems and with practical applications, as for instance fault diagnosis.

\section{References}

1. J.-P. Barbot, T. Boukhobza and M. Djemaï, "Sliding mode observer for triangular input form", IEEE Conf. on Decision and Control, Japan, 1996.

2. J.-P. Barbot, I. Belmouhoub and L. Boutat-Baddas, "Observability Normal Forms", LNCIS 295, New trends in Nonlinear dynamics and control, Springer Verlag, Ed: W. Kang et al., 2003. 
3. C. Canudas de Wit and J.J.E. Slotine, "Sliding observers in robot manipulators", Automatica, Vol. 27, No 5, pp. 859-864, 1991.

4. M. Corless and J. Tu, "State and input estimation for a class of uncertain systems", Automatica, Vol. 34, No 6, pp. 757-764, 1998.

5. J. Davila and L. Fridman, "Observation and identification of mechanical systems via second order sliding modes", in $8^{\text {th }}$ International Workshop on Variable Structure Systems, Barcelona, 2004.

6. M. Djemaï, J.P. Barbot, A. Glumineau and R. Boisliveau, "Nonlinear flux sliding mode observer", in IEEE CSCC99, IMACS, Athens, Greece, 1999.

7. S. V. Drakunov, "Sliding-mode Observer Based on Equivalent Control Method", in IEEE Conf. on Decision and Control, Tucson, Arizona, 1992.

8. S. V. Drakunov and V. I. Utkin, "Sliding mode observers. Tutorial", in IEEE Conference on Decision and Control, New-Orleans, LA, 1995.

9. B. Drazenovic, "The Invariance Conditions in Variable Structure Systems", $A u$ tomatica, Vol. 5, No 3, pp. 287-295, 1969.

10. C. Edwards and S. Spurgeon, "On the development of discontinuous observers", International Journal of Control, Vol. 59, pp. 1211-1229, 1994.

11. C. Edwards and S. Spurgeon, Sliding mode control: theory and applications, Taylor and Francis Eds, 1998.

12. C. Edwards and S. K. Spurgeon and R. J. Patton, "Sliding mode observers for fault detection and isolation", Automatica, Vol. 36, pp. 541-553, 2000.

13. C. Edwards, "A comparison of sliding mode and unknown input observers for fault reconstruction", in IEEE Conf. on Decision and Control, Bahamas, 2004.

14. C. Edwards and C. P. Tan, "Sensor fault tolerant control using sliding mode observers", Control Engineering Practice, to appear.

15. T. Floquet and J.P. Barbot, "A sliding mode approach of unknown input observers for linear systems", in IEEE Conference on Decision and Control, Bahamas, 2004.

16. T. Floquet, J.P. Barbot, W. Perruquetti and M. Djemaï, "On the robust fault detection via a sliding mode disturbance observer", International Journal of Control, Vol. 77, No 7, pp. 622-629, 2004.

17. L. Fridman and A. Levant, "Higher order sliding modes", in W. Perruquetti and J. P. Barbot (Eds), Sliding Mode Control in Engineering, Marcel Dekker, pp. 53-101, 2002.

18. K. B. Goh, S. K. Spurgeon, N. B. Jones, "Fault diagnostics using sliding mode techniques", Control Engineering Practice, Vol. 10, pp. 207-217, 2002.

19. I. Haskara, Ü. Özgüner and V. I. Utkin, "On sliding mode observers via equivalent control approach", International Journal of control, Vol. 71, No. 6, pp. 1051-1067, 1998.

20. I. Haskara and Ü. Özgüner, "Equivalent value filters in disturbance estimation and state observation", Lecture Notes in Control and Information Science, Vol. 247, pp. 167-179, 1999.

21. J. Hernandez et J-P. Barbot, "Sliding observer-based feedback control for flexible joints manipulator", Automatica, Vol. 32, No 9, pp. 1243-1254, 1996.

22. M. Hou and P. C. Müller, "Design of observers for linear systems with unknown inputs", IEEE Transactions on Automatic Control, Vol. 37, No. 6, pp. 871-875, 1992.

23. A. Levant, "Sliding order and sliding accuracy in sliding mode control", International Journal of Control, Vol. 58, No. 6, 1247-1263, 1993. 
24. A. Levant, "Robust Exact Differentiation via sliding mode technique", Automatica, Vol. 34, No. 3, pp. 379-384, 1998.

25. R. Martinez-Guerra, R. Aguilar-Lopez, A. Poznyak, "Robust sliding-mode observers for on-line reaction heat monitoring in continuous chemical reactors", ASME Journal of Dynamics Systems, Measurement \& Control, Vol. 126, No 3, pp. 473-478, 2004.

26. N. Manamanni, M. Djemaï, T. Boukhobza and N. M'Sirdi, "Nonlinear sliding observer based control for a pneumatic robot leg", Int. J. of Robotics and Automation, Vol. 16, No 2, pp.100-112, 2001.

27. W. Perruquetti and J.-P. Barbot (Editors), Sliding Mode Control in Engineering, Marcel Dekker, 2002.

28. J. J. Slotine, J. K. Hedrick, and E. A. Misawa, "On sliding observers for nonlinear systems", ASME J. Dyn. System Measurement Control, vol. 109, pp. 245-252, 1987.

29. H.J. Sussman and P.V. Kokotovic, "The peaking phenomenon and the global stabilization of nonlinear systems", IEEE Transactions on Automatic Control, Vol. 36, No 4, pp. 424-440, 1991.

30. C. P. Tan, and C. Edwards, "An LMI approach for designing sliding mode observers", International Journal of Control, Vol. 74(16), pp. 1559-1568, 2001.

31. C. P. Tan and C. Edwards, "Sliding mode observers for robust detection and reconstruction of actuator and sensor faults", Int. J. of robust and nonlinear control, Vol. 13, pp. 443-463, 2003.

32. V. I. Utkin, Sliding Modes in Control and Optimization, Berlin, Germany, Springer-Verlag, 1992.

33. V. I. Utkin, J. Guldner and J. Shi, Sliding mode control in electromechanical systems, Taylor and Francis, London, 1999.

34. B.L. Walcott and Żak, "State observation of nonlinear uncertain dynamical systems", IEEE Transactions on Automatic Control, Vol. 32, pp. 88-104, 1987.

35. Y. Xiong and M. Saif, "Sliding Mode Observer for Nonlinear Uncertain Systems", IEEE Transactions on Automatic Control, vol. 46, 2001, pp. 2012-2017. 